\title{
Interaction of insulin with Burkholderia pseudomallei may be caused by a preservative
}

\author{
Andrew J H Simpson, Vanaporn Wuthiekanun
}

\begin{abstract}
Aim-To re-examine the previously reported in vitro interaction of insulin with Burkholderia pseudomallei, in the light of a suggestion that the interaction may have resulted from the presence of the preservative $\boldsymbol{m}$-cresol in commercial preparations.

Methods-Broth culture studies of $B$ pseudomallei were performed with and without the addition of $\boldsymbol{m}$-cresol and various preparations of insulin.

Results-Growth of $\boldsymbol{B}$ pseudomallei was inhibited by $m$-cresol at the concentrations found in pharmaceutical insulin preparations, and by the insulin preparation Humulin R, but not by pure insulin. Conclusions-The results of previous experiments may have been confounded by the presence of the preservative $\boldsymbol{m}$-cresol. (F Clin Pathol 2000;53:159-160)
\end{abstract}

Keywords: Burkholderia pseudomallei, melioidosis, insulin, cresol

Insulin has been described previously as inhibitory to the growth of Burkholderia (formerly Pseudomonas) pseudomallei, the causative organism of melioidosis, both in vitro and in animal studies. ${ }^{1}$ Diabetes mellitus is the major risk factor for melioidosis in Thailand, ${ }^{2}$ and insulin deficiency might therefore contribute to this increased susceptibility. However, the previous in vitro studies were conducted using the commercially available human recombinant soluble insulin Humulin R (Lilly), which contains $m$-cresol (3-methylphenol) as a preservative (at a concentration of $2.5 \mathrm{mg} / \mathrm{ml}$ ). A more recent study with Burkholderia cepacia has shown that whereas commercial insulins containing $m$-cresol were inhibitory, purified insulin did not significantly affect growth. ${ }^{3}$ This raises the possibility that the original findings for $B$ pseudomallei resulted from the preservative rather than insulin. We therefore decided to re-examine the reported in vitro interaction of insulin with $B$ pseudomallei.

Faculty of Tropical Medicine, Mahidol University, 420/6

Rajvithi Road,

Bangkok 10400, Thailand

A J H Simpson

V Wuthiekanun

Correspondence to: Dr Simpson

email: fnajs@

diamond.mahidol.ac.th

Accepted for publication 13 September 1999

\section{Methods}

Minimum inhibitory concentrations (MIC) for $m$-cresol against $B$ pseudomallei were determined for 100 clinical isolates using an agar dilution method. ${ }^{4}$ These isolates were all obtained from patients who were admitted to Sappasitprasong Hospital, Ubon Ratchathani, northeast Thailand, during our ongoing clinical studies of melioidosis. ${ }^{5}$ All strains were isolated and identified as described previously. ${ }^{6}$ All isolates were stored at $-70^{\circ} \mathrm{C}$ in glycerol broth and subsequently resuscitated by subculture onto Columbia agar (Oxoid) at $37^{\circ} \mathrm{C}$ in air.

Insulin experiments were performed with one of the strains of $B$ pseudomallei (strain 316c) used in the original experiments. ${ }^{1}$ This is a clinical isolate obtained from a patient with septicaemic melioidosis who was admitted to Sappasitprasong Hospital.

Purified insulin (human recombinant, expressed in $E$ coli; Sigma), $m$-cresol (ICN Pharmaceuticals), and Humulin R $100 \mathrm{U} / \mathrm{ml}$, containing $m$-cresol at a concentration of 2.5 $\mathrm{mg} / \mathrm{ml}$ (Eli Lilly Asia), were obtained and stored according to the manufacturers' instructions. Experiments were conducted using Mueller-Hinton (M-H) broth (Oxoid). A range of concentrations of $m$-cresol (62.5-500 mg/l) were prepared in $\mathrm{M}-\mathrm{H}$ broths containing approximately $10^{6} \mathrm{cfu} / \mathrm{ml}$ of $B$ pseudomallei strain $316 \mathrm{c}$, prepared from an overnight broth culture. Normal saline was added to a positive control broth. All broths were then incubated at $37^{\circ} \mathrm{C}$ for 24 hours in air. Viable counts were performed on $100 \mu \mathrm{l}$ aliquots removed after mixing at 0 (before addition of $m$-cresol), 4,8 , and 24 hours, using serial (mixed) 10-fold dilutions in normal saline and subculture by a spread plate method onto Columbia agar. Plates were incubated for 48 hours in air at $37^{\circ} \mathrm{C}$ before counting. All experiments were performed in duplicate.

These experiments were repeated using $m$-cresol alone at a concentration of $250 \mathrm{mg} / \mathrm{l}$, and either pure insulin or Humulin R (equivalent to $250 \mathrm{mg} / \mathrm{l} m$-cresol) alone at final concentrations of $10 \mathrm{U} / \mathrm{ml}$. A further broth containing both pure insulin and $m$-cresol (250 $\mathrm{mg} / \mathrm{l})$ was included. Optical densities at 550 $\mathrm{nm}$ were measured spectrophotometrically (in duplicate) at each time point, as in the original experiments. ${ }^{1}$

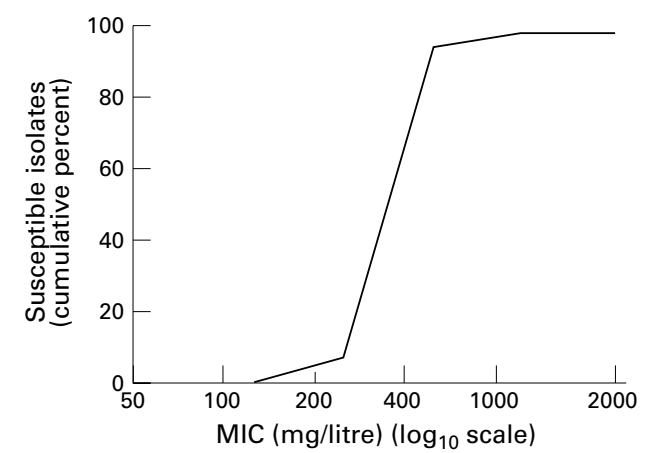

Figure 1 Cumulative percentage of Burkholderia pseudomallei isolates $(n=100)$ susceptible to increasing concentrations of $m$-cresol. 


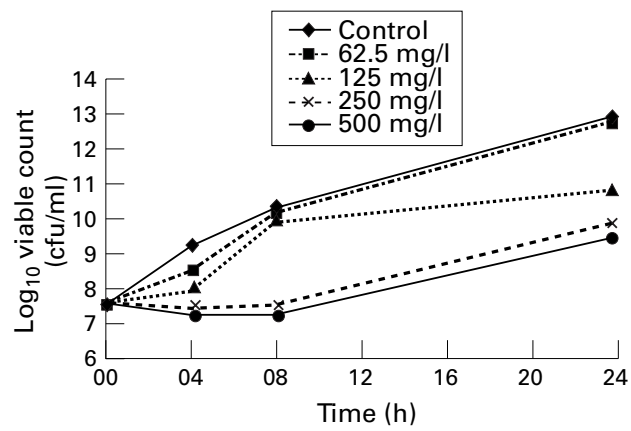

Figure 2 Effects of different concentrations of m-cresol on growth of Burkholderia pseudomallei.

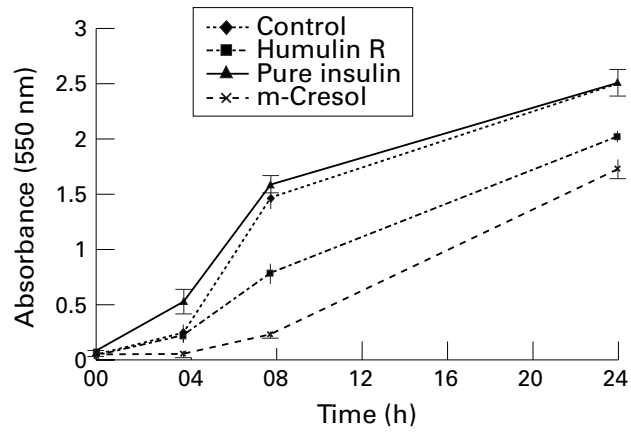

Figure 3 Optical densities during growth of Burkholderia pseudomallei in the presence of insulin or $m$-cresol. (Error bars represent $95 \%$ confidence intervals.)

\section{Results}

The $\mathrm{MIC}_{50}$ (the concentration required to inhibit the growth of $50 \%$ of isolates) and $\mathrm{MIC}_{90}$ by agar dilution for $m$-cresol against $B$ pseudomallei were both $500 \mathrm{mg} / \mathrm{l}$. The distribution of MIC results for the 100 isolates is shown in fig 1 . In broth culture, $m$-cresol inhibited growth of $B$ pseudomallei at concentrations $\geqslant 125 \mathrm{mg} / 1$ (fig 2). The effect was most marked in cultures containing 250 or $500 \mathrm{mg} / 1$ $m$-cresol.

Pure insulin did not cause significant inhibition of growth, whereas Humulin $R$ and $m$-cresol each inhibited growth of $B$ pseudomallei compared to the control (fig 3). Combined $m$-cresol/pure insulin caused similar inhibition to Humulin R (data not shown).

\section{Discussion}

Our experimental results suggest that the previously reported interaction of Humulin $\mathrm{R}$ with $B$ pseudomalle $i$ is, at least in part, caused by the presence of the $m$-cresol added as a preservative. At the experimental concentrations found in Humulin $\mathrm{R}$ at a dilution of $10 \mathrm{U} / \mathrm{ml}$, $m$-cresol $(250 \mathrm{mg} / \mathrm{l})$ caused marked inhibition of growth of $B$ pseudomallei in broth culture growth experiments. Significant inhibition also occurred with Humulin R itself and with combined insulin $/ m$-cresol. Our results therefore concur with those of Thompson and Kerr for $B$ cepacia ${ }^{3}$ and cast doubt on the reported findings for $B$ pseudomallei. ${ }^{1}$ Interestingly the effect of $m$-cresol was lessened in the presence of insulin - this requires further investigation. Of further interest is the fact that $m$-cresol at these concentrations did not ultimately prevent bacterial growth. However, the experimental concentration used $(250 \mathrm{mg} / \mathrm{l})$ was below the $\mathrm{MIC}_{50}(500 \mathrm{mg} / \mathrm{l})$, and examination of growth characteristics beyond 24 hours was outside the intended scope of these experiments.

We have not entirely disproved an interaction between insulin and $B$ pseudomallei, as these experiments were not designed to do this. Both Woods and others have demonstrated insulin binding to $B$ pseudomallei. ${ }^{17}$ The authors of the latter paper speculate that an insulin receptor forms part of a signal transfer system involving either phospholipase or protein tyrosine phosphatase, as the activities of these two enzymes were reduced in the presence of insulin. However, in conclusion, the results of previous experiments which demonstrated inhibition of growth of $B$ pseudomalle $i$ by insulin may have been confounded by the presence of the preservative $m$-cresol in Humulin R. The interaction between insulin and $B$ pseudomallei requires further investigation.

Dr Kevin Kerr originally suggested this study. We thank Paul Howe and Sayan Langla for technical assistance. This study was a part of the Wellcome-Mahidol University, Oxford Tropical Medicine Research Programme, funded by the Wellcome Trust of Great Britain. AJHS is also affiliated to the Centre for Tropical Medicine, Nuffield Department of Clinical Medicine, John Radcliffe Hospital, University of Oxford, Oxford, U.K.

1 Woods DE, Jones AL, Hill PJ. Interaction of insulin with Pseudomonas pseudomallei. Infect Immun 1993;61:404550.

2 Suputtamongkol Y, Chaowagul W, Chetchotisakd P, et al. Risk factors for melioidosis and bacteremic melioidosis. Risk factors for melioidosis and

3 Thompson S, Kerr KG. Interaction of insulin with Burkholderia cepacia. Third Conference of the Federation of Infecderia cepacia. Third Conference of the Federation of Infec-
tion Societies, Manchester, UK, 1996. Abstract P7.3. F

Infect 1997;34:180.
4 Working Party on antibiotic sensitivity testing of the British Society for Antimicrobial Chemotherapy. A guide to sensitivity testing. F Antimicrob Chemother 1991;27(suppl D):28.

5 Simpson AJH, Suputtamongkol Y, Smith MD, et al. Comparison of imipenem and ceftazidime as therapy for severe melioidosis. Clin Infect Dis 1999;29:381-7.

6 Walsh AL, Wuthiekanun V. The laboratory diagnosis of melioidosis. Br f Biomed Sci 1996;53:249-53.

7 Kanai K, Kondo E, Kurata T. Affinity and response of Burkholderia pseudomallei and Burkholderia cepacia to insulin. Southeast Asian 7 Trop Med Public Health 1996;27: 584-91. 